\title{
Covered stents for the treatment of life-threatening cervical esophageal anastomotic leaks
}

\author{
Amitabh Chak, MD, ${ }^{\mathrm{a}}$ Rohit Singh, BA, ${ }^{\mathrm{b}}$ and Philip A. Linden, MD, ${ }^{\mathrm{b}}$ Cleveland, Ohio
}

Esophagectomy with a cervical anastomosis is associated with a leak rate of approximately $10 \%$ to $20 \%$. These leaks are usually managed by drainage and are allowed to heal over several days to weeks. Rarely, a cervical anastomotic leak may drain into the chest and cause a life-threatening infection. In such instances, repair of the leak is often not possible, and takedown of the gastric conduit is necessary. After several weeks to months of recovery, reoperation is required to restore gastrointestinal continuity. Restoration of gastrointestinal continuity generally requires a major reoperation rerouting the gastric conduit through the left side of the chest or use of a colon interposition. We describe the use of a temporary fully covered silastic expandable metallic esophageal stent to treat cervical leaks that otherwise would have required takedown of the conduit.

\section{RESULTS}

The first patient was a 60-year-old man with a history of Crohn disease and prior total colectomy with end ileostomy. He had a T2 N0 adenocarcinoma of the gastroesophageal junction and underwent a McKeown tri-incisional esophagectomy. On a routine postoperative swallow he was seen to have a large posteriorly based leak at the anastomosis, $5 \mathrm{~cm}$ beyond the cricopharyngeus, into the mediastinum (Figure 1). Drains were placed into this space through the cervical incision. The patient did well initially until signs of sepsis developed and an enlarging right-sided effusion was seen. The effusion was purulent and the patient was brought to the operating room where he underwent drainage of the empyema with decortication. On esophagoscopy, the posterior half of the anastomosis was seen to be interrupted. A covered Alimaxx-E (Alveolus Inc, Charlotte, NC) esophageal stent, $22 \mathrm{~mm}$ in diameter and $100 \mathrm{~mm}$ in length, was deployed across the anastomosis (Figure 2). After stent deployment, the patient's condition improved. He underwent

\footnotetext{
From the Division of Gastroenterology ${ }^{\mathrm{a}}$ and Division of Thoracic and Esophageal Surgery, ${ }^{\mathrm{b}}$ Case Medical Center/University Hospitals, Case Western Reserve School of Medicine, Cleveland, Ohio.

Disclosures: Authors have nothing to disclose with regard to commercial support.

Received for publication June 3, 2010; revisions received June 30, 2010; accepted for publication July 12, 2010; available ahead of print Aug 16, 2010.

Address for reprints: Philip A. Linden, MD, Case Medical Center, Thoracic and Esophageal Surgery, 11100 Euclid Ave, Cleveland, OH 44106-5011 (E-mail: philip.linden@uhhospitals.org).

J Thorac Cardiovasc Surg 2011;141:843-4

$0022-5223 / \$ 36.00$

Copyright (c) 2011 by The American Association for Thoracic Surgery doi:10.1016/j.jtcvs.2010.07.019
}

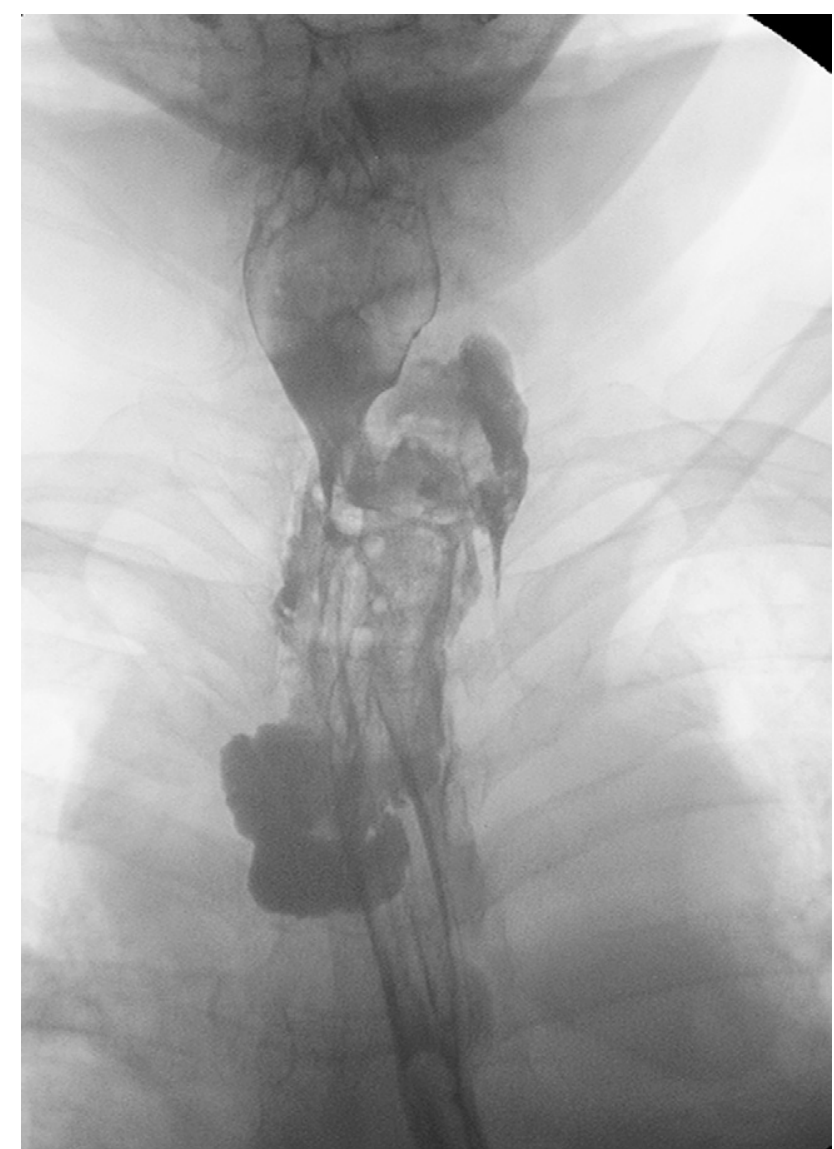

FIGURE 1. A large amount of contrast medium is seen pooling in the posterior mediastinum.

a repeat swallow and was seen to have a small persistent leak, which was drained by a Blake drain placed in the superior posterior mediastinum at the time of decortication. He was maintained on jejunal tube feedings, was discharged from the hospital, and the stent was removed 6 weeks later. A swallow showed no residual leak and the patient resumed an oral diet. The patient's swallowing is nearly normal 2 years after the procedure.

The second patient was a 70-year-old man with a T1 N0 adenocarcinoma of the gastroesophageal junction in the setting of Barrett's esophagus. He underwent a McKeown esophagectomy employing a thoracoscopic dissection. Six days after the operation he was noted to have redness and swelling around the cervical incision, which was opened. Several days later signs of systemic infection developed, 


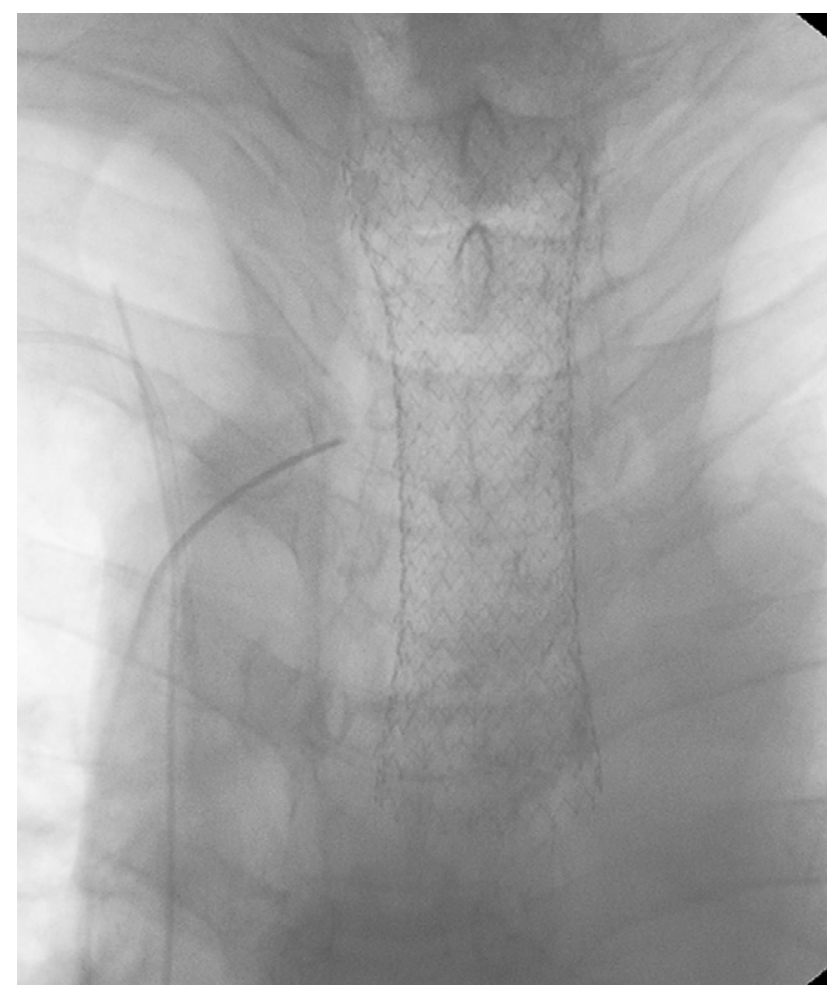

FIGURE 2. Radiograph of stent across the anastomosis.

and a swallow study showed leakage of contrast material into the right side of the chest from the area of the cervical anastomosis, which was $6 \mathrm{~cm}$ beyond the cricopharyngeus. He was taken to the operating room where he underwent video-assisted thoracic surgical drainage and endoscopic placement of a covered Alimaxx E stent $22 \mathrm{~mm}$ in diameter and $100 \mathrm{~mm}$ in length. A postoperative swallow showed no leak. The patient's fever, leukocytosis, and systemic sepsis resolved. The patients was sent home on tube feeds and clear sips and returned for stent removal 4 weeks later. After stent removal, a small endoscopic diverticulum was noted on endoscopy, and the stent was replaced. One week later the stent had migrated into the stomach, and it was removed and not replaced. A swallow study showed no leak. The patient had drainage of saliva out of his neck approximately 1 week later, and a swallow study at that time showed leakage through the cervical incision, but no leakage into the chest.
His neck leak subsequently healed and he is without dysphagia 4 months later.

\section{DISCUSSION}

Esophageal stents have recently been described in the setting of esophageal anastomotic leak and perforation. Hunerbein and associates ${ }^{1}$ originally described the use of the silastic Polyflex stent (Boston Scientific, Natick, Mass) for the treatment of 9 consecutive intrathoracic anastomotic leaks after esophagectomy. Immediate leak occlusion was obtained in 8 of 9 patients; 2 of the stents migrated. The stents were removed after an average of 29 days. Freeman, Van Workom, and Ascioti ${ }^{2}$ described the use of Polyflex stents in 18 patients with acute iatrogenic perforations of the intrathoracic esophagus with an $18 \%$ migration rate and a leak occlusion rate of $94 \%$ as determined by esophagogram. Patients had the stents removed after a mean of 52 days. Recent publications have described the use of fully coated self-expanding metallic stents in the treatment of benign perforations, but excluded postoperative anastomotic leaks from consideration. ${ }^{3}$

Although life-threatening leaks after a cervical esophageal anastomosis are rare, they are typically difficult to repair surgically owing to the tight constraints of the thoracic inlet and the limited mobility of the esophageal conduit. Stent placement provides an alternative to takedown of the conduit in these situations. The experience in these $2 \mathrm{pa}-$ tients shows the limitations of these stents: migration, incomplete leak sealing, and failure of complete healing after stent removal. Nonetheless, the use of covered esophageal stents allowed for the preservation of esophageal continuity and reversal of sepsis in these patients who had a cervical esophageal anastomotic leak into the chest.

\section{References}

1. Hunerbein M, Stroszczynski C, Moesta KT, Schlag PM. Treatment of thoracic anastomotic leaks after esophagectomy with self-expanding plastic stents. Ann Surg. 2004;240:801-7.

2. Freeman RK, Van Workom JM, Ascioti AJ. Esophageal stent placement for the treatment of iatrogenic intrathoracic esophageal perforation. Ann Thorac Surg. 2007;83:2003-8

3. van Heel NC, Haringsma J, Spaander MC, Bruno MJ, Kuipers EJ. Short-term esophageal stenting in the management of benign perforations. Am J Gastroenterol. 2010;105:1515-20. 